\title{
Correlação entre características antropométricas individuais e as medidas da fóvea tibial do ligamento cruzado anterior*
}

\section{Correlation bettween Individual Anthropometric Characteristics and Anterior Cruciate Ligament Tibial Fovea Measurements}

\author{
Julio Cesar Gali1,20 David Braz Del Giglio ${ }^{1,2}$ Luís Fernando Patriarcha ${ }^{1,2}$ Bruno Azi Pacileo Cruz ${ }^{1,2}$ \\ Julio Cesar Gali Filho ${ }^{3}$ \\ ${ }^{1}$ Departamento de Ortopedia, Faculdade de Ciências Médicas e da Saúde, \\ Pontifícia Universidade Católica de São Paulo, Sorocaba, SP, Brasil \\ 2 Serviço de Cirurgia de Joelho, Hospital Santa Lucinda, Sorocaba, SP, Brasil \\ Endereço para correspondência Julio Cesar Gali, PhD, Rua Caracas \\ ${ }^{3}$ Núcleo de Ortopedia e Traumatologia do Esporte, Sorocaba, SP, Brasil \\ 418, Sorocaba, SP, 18046-718, Brasil (e-mail: juliogali@note.med.br). \\ Rev Bras Ortop 2020;55(1):88-94.
}

\begin{abstract}
Resumo
Palavras-chave

- tíbia

- ligamento cruzado anterior

- anatomia

Objetivo Avaliar possíveis relações entre o peso e altura de pacientes submetidos à artroplastia total do joelho com o comprimento, largura e área da fóvea do ligamento cruzado anterior, verificados na cirurgia.

Métodos Um total de 33 superfícies articulares tibias proximais, obtidas nos cortes tibiais de artroplastia total do joelho de 33 pacientes, foram utilizadas no presente estudo. O ligamento cruzado anterior foi dissecado cuidadosamente e ressecado com bisturi delicado, para expor sua fóvea. Depois, a periferia dessa fóvea foi demarcada por pequenos pontos, com um marcador. Cada peça foi fotografada e as medições do comprimento, largura e área da fóvea tibial do ligamento cruzado anterior foram feitas com o programa ImageJ (National Institutes of Health, Bethesda, MD, EUA). A análise estatística avaliou a correlação entre os dados antropométricos dos pacientes com as medidas da fóvea tibial do ligamento cruzado anterior.

Resultados O comprimento, a largura e a área médios da fóvea tibial do ligamento cruzado anterior foram, respectivamente $11,7 \pm 2,0 \mathrm{~mm}, \quad 7,1 \pm 1,4 \mathrm{~mm} \quad$ e $151,3 \pm 22,2 \mathrm{~mm}^{2}$. Houve relação estatisticamente significativa entre a altura dos pacientes e a largura da fóvea tibial do ligamento cruzado anterior. A largura da fóvea do ligamento cruzado anterior pôde ser predita pela fórmula: largura $=107,294-$ $(133,179 \times$ altura $)+(44,009 \times$ altura ao quadrado $)$.

Conclusão A altura dos pacientes pôde a prever a largura da fóvea tibial do ligamento cruzado anterior e, assim, pode ajudar os cirurgiões escolher o enxerto mais adequado para cada paciente, nas reconstruções do ligamento cruzado anterior.
\end{abstract}

\footnotetext{
* Trabalho feito no Departamento de Ortopedia da Faculdade de Ciências Médicas e da Saúde da Pontifícia Universidade Católica de São Paulo, Sorocaba, SP, Brasil.
}

recebido

17 de Agosto de 2018 aceito

16 de Outubro de 2018
DOI https://doi.org/ 10.1055/s-0039-1700816. ISSN $0102-3616$.
Copyright $(\underset{0}{2020}$ by Sociedade Brasileira License terms de Ortopedia e Traumatologia. Published by Thieme Revinter Publicações Ltda, Rio de Janeiro, Brazil 
Abstract

\section{Keywords \\ - tibia \\ - anterior cruciate ligament \\ - anatomy}

Objective To evaluate possible connections between the weight and height of patients submitted to total knee arthroplasty (TKA), with the length, width and area of the anterior cruciate ligament ( $\mathrm{ACL}$ ) fovea, as verified during surgery.

Methods A total of 33 proximal tibial joint surfaces, obtained from TKA tibial sections of 33 patients, were used in the present study. The ACL was resected with a delicate scalpel to expose the ACL tibial fovea. Then the periphery of this fovea was delimited with a marker pen by means of small dots. Each piece was photographed, and the ACL tibial fovea length, width, and area were measured with the ImageJ (National Institutes of Health, Bethesda, MD, USA) software. Statistical analysis studied the correlation between anthropometrics data of the patients and the measurements of the ACL tibial fovea.

Results The ACL tibial fovea length, width, and area were, respectively, $11.7 \pm 2.0 \mathrm{~mm}, 7.1 \pm 1.4 \mathrm{~mm}$ and $151.3 \pm 22.2 \mathrm{~mm}^{2}$. There was a statistically significant relationship between the height of the patients and the width of the $A C L$ tibial fovea. The width of the ACL fovea could be predicted by the formula: width $=107.294-(133.179 \times$ height $)+(44.009 \times$ squared height $)$.

Conclusion The height of the patients may predict the width of the ACL tibial fovea, and therefore, may allow surgeons to choose the more adequate graft for each patient in $\mathrm{ACL}$ reconstruction.

\section{Introdução}

O número de reconstruções do ligamento cruzado anterior (LCA), no período compreendido entre 1994 e 2006, teve um aumento de $50 \%$, especialmente em mulheres e em pessoas com $<20$ anos e $>40$ anos de idade. ${ }^{1} \mathrm{O}$ resultado da reconstrução do LCA depende da resposta biológica do paciente, da técnica cirúrgica e da reabilitação. A tendência atual é realizar de reconstruções anatômicas, definidas como aquelas com a intenção de buscar a restauração funcional do LCA às suas dimensões nativas, orientação do colágeno e sítios de inserção, a fim de tentar reproduzir a anatomia normal, restabelecer a cinemática e proporcionar saúde articular em longo prazo. ${ }^{2}$

Uma concepção mais recente é de que as reconstruções necessitam ser individualizadas. Como não existem dois LCAs iguais, o enxerto deve ser moldado para cada indivíduo. ${ }^{3}$ Uma vez que a reconstrução do LCA pode ser considerada um transplante de enxerto, o tamanho deste deve ser especialmente avaliado. Entretanto, na maioria das cirurgias reconstrutivas que usam enxertos autólogos, o tamanho do LCA é determinado principalmente pelas dimensões do enxerto obtido e não pela medida da inserção do LCA. Se o cirurgião puder prever o tamanho da inserção tibial do LCA antes da cirurgia, também poderá escolher o tamanho mais adequado do autoenxerto. ${ }^{4}$

Portanto, antecipar as dimensões da inserção do LCA pode ser valoroso para melhor planejamento cirúrgico. Isso pode ser feito por tomografia computadorizada (TC $)^{5,6}$ ou ressonância magnética (RM), ${ }^{7-11}$ mas também pode ser previsto pelas características físicas individuais. ${ }^{3,4}$

Nesta década, alguns autores procuraram correlacionar o peso e altura dos pacientes com o tamanho de sua inserção tibial do LCA. Esses estudos foram feitos nos Estados Unidos ${ }^{3} \mathrm{e}$ na Coreia do Sul. ${ }^{11-13}$ Portanto, é importante um estudo para avaliar se existe alguma relação entre o peso e a altura dos pacientes e as dimensões da fóvea tibial do LCA na população brasileira, composta de uma miscigenação de etnias, particularmente a africana, portuguesa, italiana, indígena nativa, espanhola, alemã, japonesa, polonesa, árabe e judaica. ${ }^{14}$

O objetivo do presente trabalho foi avaliar possíveis relações entre o peso e a altura de pacientes submetidos à artroplastia total do joelho (ATJ) com o comprimento, largura e área de fóvea tibial do LCA verificadas na ATJ. Nossa hipótese foi que a avaliação pré-operatória das características antropométricas de nosso pacientes poderia antecipar as dimensões da fóvea tibial do LCA.

\section{Métodos}

Obtivemos a aprovação do Comitê de Ética de nossa instituição para a realização deste estudo (CAAE: 21220013.6.0000.5373, Parecer: 509.126). As informações do peso e altura dos pacientes foram conseguidas nos prontuários do Hospital da Instituição onde o trabalho foi realizado.

De setembro de 2014 a junho de 2017, foram realizadas 74 ATJs em nosso serviço. Somente foram incluídos em nossa pesquisa os joelhos cujos LCAs apresentavam aspecto macroscópico normal. Foram considerados fatores de exclusão: história de trauma articular pregresso, doenças infecciosas ou inflamatórias que pudessem alterar a inserção tibial do LCA, alterações morfológicas macroscópicas do LCA e presença de osteófitos ao redor da inserção tibial do LCA, no momento da avaliação. Nos casos de cirurgias bilaterais, apenas um joelho (direito ou esquerdo) foi escolhido, por sorteio (cara ou coroa). Assim, 41 joelhos foram excluídos de nossa investigação, 7 $(9,4 \%)$ por serem de pacientes que tiveram artroplastia bilateral e 34 por alterações macroscópicas da anatomia normal do LCA ou pela presença de osteófitos que alteraram a inserção do $\operatorname{LCA}(45,9 \%)$. 


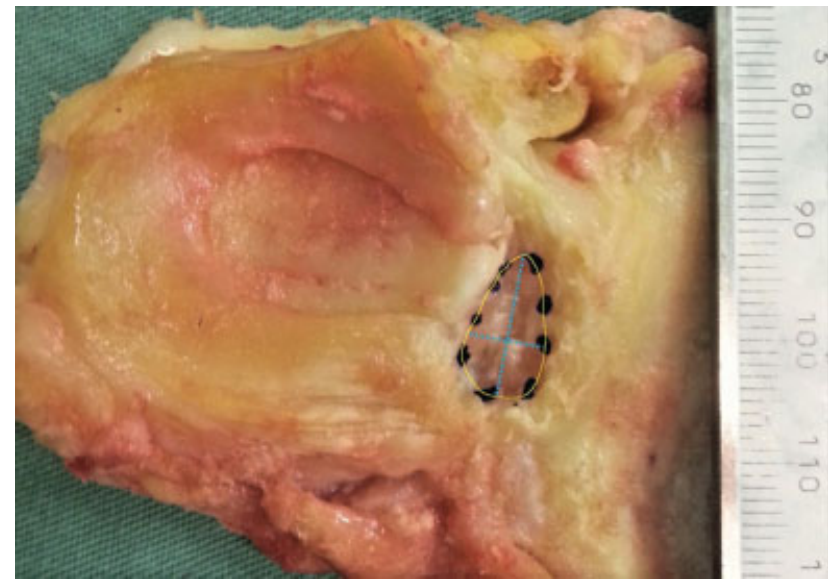

Fig. 1 Visão superior da face articular superior tibial, retirada na artroplastia, com a delimitação da fóvea do LCA e a definição de seu comprimento e largura. Fonte: Arquivo cedido pela instituição.

Deste modo, nossa amostra final foi constituída por 33 joelhos de 33 pacientes. Dezenove eram mulheres (57,6\%) e 14 , homens (42,4\%). Dezoito joelhos eram direitos (54,5\%) e 15 , esquerdos (45,5\%). A idade média dos indivíduos foi de 69,5 anos $( \pm 7,3)$, o peso mediano foi de $78,4 \mathrm{~kg}$ $( \pm 12,3)$ e altura média foi de $1,58 \mathrm{~m}( \pm 0,08)$.

Cada peça, correspondente à porção tibial proximal, retirada no corte tibial das ATJ, foi avaliada pelo mesmo cirurgião, sênior da equipe, após a cirurgia, com lente magnificadora de quatro vezes. Após dissecação minuciosa do LCA, este foi ressecado, com bisturi delicado, para expor sua fóvea tibial, uma depressão entre as eminências intercondilares correspondente à inserção tibial do LCA. $^{5}$ A fóvea foi delimitada, em sua periferia, através de pequenos pontos, com caneta marcadora. Uma régua milimetrada foi colocada ao lado das peças, para servir de parâmetro das medições e, em seguida, cada peça foi fotografada, com uma máquina fotográfica digital Nikon D3100 (Nikon, Tóquio, Japão), que foi posicionada paralela ao plano da mesa de exame das peças.

Todas as medições foram feitas posteriormente, utilizandose o programa ImageJ (National Institutes of Health, Bethesda, MD, EUA), por outro membro da equipe. O comprimento da fóvea tibial do LCA foi definido como seu maior eixo na direção anteroposterior e, sua largura, como seu maior eixo na direção lateromedial, perpendicular ao eixo anteroposterior. Para se medir a área, foi feita, no computador, a união dos pontos previamente demarcados ( - Figura 1). Obtivemos, também, a razão entre o comprimento e a largura da fóvea tibial do LCA. As medidas foram feitas duas vezes e foi utilizada a média dos valores obtidos.

\section{Análise Estatística}

Utilizamos a análise multivariada de variância (MANOVA, na sigla em inglês) para a interpretação dos resultados, tendo em vista que a amostra pode ser considerada pequena e tem três variáveis dependentes (largura, comprimento e área). Dessa forma, adotamos como estratégia a aplicação de uma MANOVA de um fator (gênero) e duas covariáveis (altura e peso) e, simultaneamente com a MANOVA, executamos os cálculos dos intervalos de confiança e comparações múltiplas ajustadas por bootstrap ( $n=1.000$; com correção de viés) para apontar com precisão onde estavam as diferenças significantes.

Para exame da normalidade de cada uma das variáveis foi usado o teste Shapiro-Wilk, com nível de 5\% de significância, e rejeitamos a normalidade univariada em todas as variáveis dependentes, exceto comprimento. $O$ teste de Levene foi feito para os gêneros e lados e, em todas as variáveis, não rejeitamos a hipótese de homogeneidade das variâncias (-Tabela $\mathbf{1}$ ).

\section{Resultados}

O comprimento, largura e área médios da fóvea tibial do LCA foram, respectivamente, $11,7 \pm 2,0 \mathrm{~mm} ; 7,1 \pm 1,4 \mathrm{~mm}$ e $151,3 \pm 22,2 \mathrm{~mm}^{2}$. Na média, o comprimento da fóvea do LCA foi 1,64 vezes o tamanho da largura. Em 27 joelhos $(81,8 \%)$ o comprimento da fóvea tibial do LCA foi $<14 \mathrm{~mm}$ e, em 28 pacientes $(84,8 \%)$, a largura da fóvea foi $<8 \mathrm{~mm}$. A íntegra dos resultados encontra-se descrita na - Tabela 2.

A totalidade das correlações individuais foram significativas; entretanto, nenhum dos coeficientes superou o valor de 0,90 , limite da multicolinearidade. Encontramos relação estatisticamente significante apenas entre a altura dos pacientes e a largura da fóvea tibial do LCA. A relação entre essas duas variáveis encontra-se na - Figura 2. Nesta, existe um valor sobressalente que, no entanto, não nos pareceu ser

Tabela 1 Homogeneidade e Normalidade da Amostra

\begin{tabular}{|l|l|l|l|l|l|l|}
\hline & \multicolumn{2}{|l|}{ Levene $^{*}$} & \multicolumn{2}{l|}{ Shapiro-Wilk } \\
\hline & Estatistica & gl & Sig. & Estatistica & gl & Sig. \\
\hline Idade & 0,686 & 31 & 0,414 & 0,957 & 33 & 0,208 \\
\hline Peso & 0,173 & 31 & 0,68 & 0,973 & 33 & 0,554 \\
\hline Altura & 2,337 & 31 & 0,136 & 0,928 & 33 & 0,030 \\
\hline Largura & 0,929 & 31 & 0,343 & 0,850 & 33 & 0,000 \\
\hline Comprimento & 0,437 & 31 & 0,514 & 0,977 & 33 & 0,680 \\
\hline Área & 1,967 & 31 & 0,171 & 0,831 & & 33 \\
\hline
\end{tabular}

Abbreviation: GL,graus de liberdade (tamanho da amostra- o número de parãmetros estimados).

Fonte: Arquivo cedido pela instituição.

*Variável de agrupamento: gênero. 
Tabela 2 Distribuição dos pacientes e de todos os resultados

\begin{tabular}{|c|c|c|c|c|c|c|c|c|}
\hline PACIENTE & IDADE & GÊNERO & PESO & ALTURA & LADO & LARGURA & COMPRIMENTO & ÁREA \\
\hline & & & $\mathrm{Kg}$ & $\mathrm{m}$ & & $\mathrm{mm}$ & $\mathrm{mm}$ & $\mathrm{mm}^{2}$ \\
\hline 1 & 69 & $\mathrm{~F}$ & 51 & 1,48 & $\mathrm{D}$ & 5,96 & 10,27 & 50,4 \\
\hline 2 & 72 & $M$ & 73 & 1,62 & $E$ & 6,29 & 10,10 & 52,8 \\
\hline 3 & 60 & $M$ & 79 & 1,65 & $E$ & 7,55 & 14,27 & 78,9 \\
\hline 4 & 67 & $\mathrm{~F}$ & 80 & 1,58 & $\mathrm{D}$ & 7,03 & 12,16 & 61,8 \\
\hline 5 & 66 & $\mathrm{~F}$ & 77 & 1,53 & $\mathrm{E}$ & 7,92 & 11,92 & 67,1 \\
\hline 6 & 72 & $M$ & 108 & 1,64 & $E$ & 6,48 & 9,70 & 49,4 \\
\hline 7 & 75 & $\mathrm{~F}$ & 80 & 1,50 & $\mathrm{D}$ & 5,09 & 9,56 & 35,8 \\
\hline 8 & 68 & $\mathrm{~F}$ & 85 & 1,57 & $\mathrm{D}$ & 6,76 & 9,52 & 49,2 \\
\hline 9 & 81 & $M$ & 70 & 1,70 & $\mathrm{D}$ & 7,22 & 10,97 & 52,3 \\
\hline 10 & 79 & $\mathrm{~F}$ & 69 & 1,50 & $E$ & 6,99 & 11,38 & 51,2 \\
\hline 11 & 81 & $M$ & 70 & 1,70 & $E$ & 6,11 & 9,44 & 51,3 \\
\hline 12 & 62 & $\mathrm{~F}$ & 77 & 1,56 & $E$ & 7,87 & 14,62 & 88,2 \\
\hline 13 & 62 & $M$ & 76 & 1,78 & $D$ & 8,75 & 15,86 & 90,8 \\
\hline 14 & 58 & $\mathrm{~F}$ & 72 & 1,50 & $D$ & 6,96 & 10,13 & 47,7 \\
\hline 15 & 74 & $M$ & 81 & 1,60 & $\mathrm{D}$ & 8,33 & 12,98 & 76,6 \\
\hline 16 & 64 & $M$ & 71 & 1,62 & $D$ & 7,52 & 11,68 & 68,0 \\
\hline 17 & 70 & $\mathrm{~F}$ & 94 & 1,58 & $E$ & 7,60 & 12,65 & 70,4 \\
\hline 18 & 83 & $\mathrm{~F}$ & 89 & 1,55 & $E$ & 7,13 & 10,85 & 51,0 \\
\hline 19 & 62 & $\mathrm{~F}$ & 84 & 1,60 & $\mathrm{D}$ & 7,00 & 10,75 & 52,9 \\
\hline 20 & 58 & $M$ & 88 & 1,64 & $E$ & 8,46 & 12,20 & 66,5 \\
\hline 21 & 72 & $\mathrm{~F}$ & 63 & 1,50 & $E$ & 5,13 & 8,62 & 33,4 \\
\hline 22 & 62 & $\mathrm{~F}$ & 62 & 1,49 & $\mathrm{D}$ & 6,96 & 13,26 & 68,9 \\
\hline 23 & 60 & $M$ & 92 & 1,64 & $D$ & 7,32 & 11,89 & 61,9 \\
\hline 24 & 68 & $M$ & 66 & 1,58 & $\mathrm{D}$ & 8,16 & 14,10 & 96,6 \\
\hline 25 & 73 & $\mathrm{~F}$ & 60 & 1,50 & $E$ & 4,87 & 10,69 & 40,0 \\
\hline 26 & 71 & $M$ & 84 & 1,58 & $D$ & 6,56 & 11,54 & 56,2 \\
\hline 27 & 65 & $\mathrm{~F}$ & 90 & 1,55 & $\mathrm{D}$ & 4,98 & 13,11 & 54,0 \\
\hline 28 & 66 & $\mathrm{~F}$ & 73 & 1,45 & $\bar{E}$ & 7,13 & 11,84 & 66,0 \\
\hline 29 & 77 & $M$ & 76 & 1,64 & $\mathrm{D}$ & 6,23 & 13,13 & 60,1 \\
\hline 30 & 64 & $\mathrm{~F}$ & 108 & 1,60 & $E$ & 7,34 & 10,00 & 53,7 \\
\hline 31 & 73 & $\mathrm{~F}$ & 77 & 1,55 & $\mathrm{D}$ & 5,90 & 8,06 & 37,3 \\
\hline 32 & 83 & $\mathrm{~F}$ & 75 & 1,53 & D & 7,74 & 14,51 & 84,1 \\
\hline 33 & 79 & $M$ & 88 & 1,85 & $E$ & 12,80 & 16,69 & 151,3 \\
\hline MÍNIMO & 58,00 & $\mathrm{H} 14$ & 51,00 & 1,45 & D 18 & 4,87 & 8,06 & 33,40 \\
\hline MÁXIMO & 83,00 & M 19 & 108,00 & 1,85 & E 15 & 12,80 & 16,69 & 151,30 \\
\hline MÉDIA & 69,58 & & 78,42 & 1,59 & & 7,10 & 11,77 & 62,90 \\
\hline DESVIO PADRÃO & 7,391001 & & 12,36434 & 0,086446 & & 1,430719 & 2,052747789 & 22,29646111 \\
\hline
\end{tabular}

Fonte: Arquivo cedido pela instituição.

um valor extremo (outlier), tendo em vista que uma altura de $1,85 \mathrm{~m}$ é perfeitamente factível. Existiu uma relação quadrática entre altura e largura, a qual se ajustou muito melhor do que uma relação linear (-Figura $\mathbf{3}$ ).

O ajuste da reta desta tabela por Mínimos Quadrados Ordinários (MQO) retornou $\mathrm{um} \mathrm{R}^{2}$ ajustado de 0,369 , enquanto que o ajuste da curva quadrática retornou um valor de $\mathrm{R}^{2}$ ajustado igual a 0,471 . 0 valor do $\mathrm{R}^{2}$ desse modelo foi de 0,71 , indicando que $71 \%$ da variação da largura da fóvea tibial do LCA pode ser explicada pela variação da altura do paciente. Os valores dos coeficientes do modelo quadrático, todos significativos ao nível de $5 \%$ de confiança, foram: Largura $=107,294-(133,179 \times$ altura $)+(44,009 \times$ altura ao quadrado). 


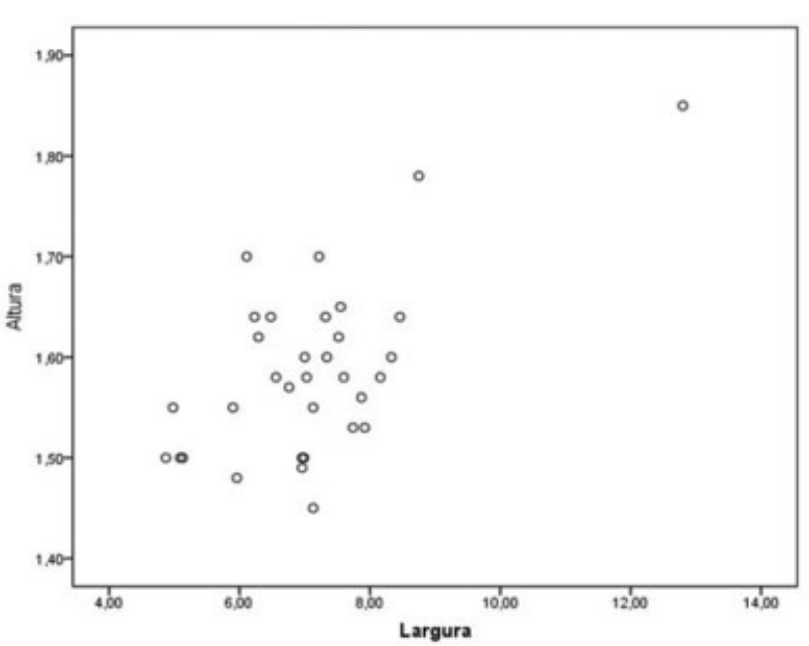

Fig. 2 Relação entre as variáveis altura dos pacientes e a largura da inserção tibial do LCA. Fonte: Arquivo cedido pela instituição.

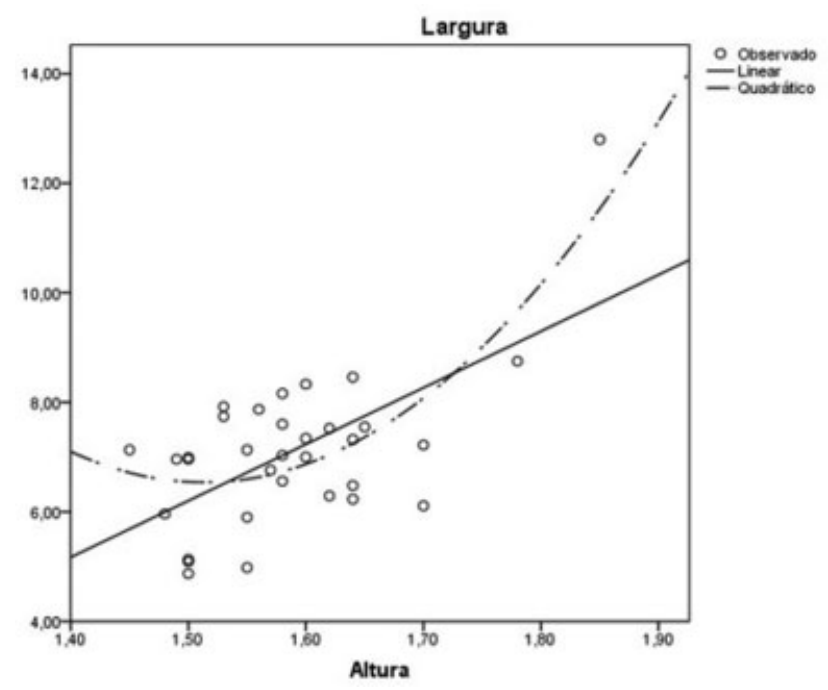

Fig. 3 Relação quadrática entre as variáveis altura dos pacientes e a largura da inserção tibial do LCA. Fonte: Arquivo cedido pela instituição.

\section{Discussão}

O principal achado de nosso estudo foi que a largura da fóvea tibial do LCA, verificada em joelhos de indivíduos submetidos a ATJ, foi relacionada com a altura do paciente. Em $45,9 \%$ da nossa casuística inicial, havia alterações macroscópicas da anatomia normal do LCA ou presença de osteófitos ao redor da inserção desse ligamento. Douglas et $a{ }^{1},{ }^{15} \mathrm{em} 95$ pacientes submetidos a ATJ, constataram ausência do LCA em 12\% dos pacientes e anormalidades macroscópicas em $45,2 \%$ dos indivíduos. Lee et $\mathrm{al}^{16}$ avaliaram a integridade do LCA em 107 joelhos submetidos à ATJ. Em 41 pacientes (39\%) o ligamento estava deficiente no momento da cirurgia, sendo que $31 \%$ destes podiam se lembrar de episódio consistente com uma lesão do LCA ou história de instabilidade pregressa. Para Mullaji et al, ${ }^{17} 40 \%$ dos joelhos com artrite, no momento da artroplastia, mostraram o LCA severamente degenerado.
Em 27 joelhos $(81,8 \%)$ de nossa casuística, o comprimento da fóvea do LCA foi $<14 \mathrm{~mm}$ e, em 28 pacientes $(84,8 \%)$ sua largura foi $<8 \mathrm{~mm}$. Em nossa metodologia, ressecamos a porção tibial do LCA e medimos sua fóvea. Esse método de avaliação diferiu da maioria das pesquisas encontradas na literatura, onde a área de inserção tibial do LCA foi medida delineando sua periferia, o que engloba também expansões de sua fixação. Por isso mesmo nossas medidas são muito semelhantes àquelas obtidas por Purnell et $\mathrm{al}^{5}$ e menor que as dos demais autores. Outra possível explicação para nossas medidas serem menores que as de outros autores foi que a altura de nossos pacientes foi relativamente baixa $(1,59 \pm 0,08 \mathrm{~m})$ e, como a largura da fóvea do LCA foi relacionada à altura do pacientes, sua medida também foi menor. A comparação entre nossos resultados e de outros autores, pelos vários modos de avaliação, encontra-se na - Tabela 3. ${ }^{3-13,18-24}$

De fato, Lord et a ${ }^{25}$ publicaram um estudo da função das fibras insercionais tibiais do LCA na resistência dos deslocamentos anterior e rotacional, com robótica, em seis graus de liberdade de movimento. Concluíram que as fibras centrais, posteriores e laterais da inserção tibial do LCA têm um papel secundário na restrição dos deslocamentos tibiais e que as fibras mais importantes são as anteriores e a porção periférica anteromedial. Entretanto, não podemos ter certeza de que isso aconteça in vivo, dada ao conjunto de interações dos restritores estáticos e dinâmicos responsáveis pela estabilização do joelho nos diversos movimentos tridimensionais simultâneos que acontecem durante as atividades diárias do ser humano.

A nosso ver, o enxerto é diferente do ligamento original, portanto não precisa, necessariamente, reproduzir as mesmas medidas desse ligamento mas, sim, apresentar força para falha e rigidez semelhantes ao ligamento primário. Em nosso estudo, o comprimento da inserção do LCA foi 1,64 vezes o tamanho da largura, portanto esta última é o fator limitante na determinação do diâmetro do enxerto a ser utilizado.

Hamada et $\mathrm{al}^{26}$ reportaram que o enxerto não deve cobrir toda a área de inserção ligamentar, tendo em vista que estes apresentam propriedades mecânicas maiores que o LCA nativo e que se tornam hipertróficos após o implante. Por outro lado LaPrade et $\mathrm{al}^{27}$ acreditam que a previsão da largura da inserção tibial do LCA tem sido negligenciada e que enxertos grandes podem lesar as raízes anterior ou posterior do menisco lateral.

Nossas medidas foram feitas em joelhos de pacientes, durante ATJ. Houve correlação positiva entre a altura do paciente e a largura da fóvea tibial do LCA. Kopf et al ${ }^{3}$ quantificaram as medidas das inserções do LCA em 137 pacientes durante reconstruções do LCA. O comprimento médio da inserção tibial do LCA foi entre 16 e $18 \mathrm{~mm}$ em $66,4 \%$ dos indivíduos. Houve correlação positiva significante entre o peso e altura dos pacientes e o comprimento da inserção tibial do LCA $(p<0.05)$. No entanto, a delimitação da inserção do tibial, na artroscopia, foi feita com eletrocautério e as medidas feitas com uma régua, o que pode gerar um viés de subjetividade.

Ichiba et $\mathrm{al}^{7}$ avaliaram as medidas da inserção tibial do LCA em 100 RMs de 100 pacientes, 50 homens e 50 mulheres. Para esses autores, o comprimento médio dessa inserção, nos homens, foi de $16,1 \mathrm{~mm}$ e, nas mulheres, $14,3 \mathrm{~mm}$ (média de $15,2 \pm 1,9 \mathrm{~mm}$ ). Houve correlação significante entre o 
Tabela 3 Descrição das diversas medidas da inserção tibial do LCA, autores, ano de publicação, referência e o método de avaliação

\begin{tabular}{|c|c|c|c|c|}
\hline Referência & Comprimento (mm) & Largura $(\mathrm{mm})$ & Razão C/L & Área $\left(\mathrm{mm}^{2}\right)$ \\
\hline Morgan et al ${ }^{18}(1995)^{a}$ & 18 & 10 & 1,8 & NA \\
\hline Park et al ${ }^{12}(2015)^{a}$ & 13,8 & 9,8 & 1,4 & NA \\
\hline Park et al ${ }^{13}(2017)^{a}$ & 14,3 & 10,2 & 1,4 & NA \\
\hline Tállay et al ${ }^{19}(2008)^{a}$ & $19,5 \pm 2,6$ & $10,3 \pm 1,9$ & 1,89 & NA \\
\hline Kim et $\mathrm{al}^{11}(2018)^{\mathrm{a}}$ & 13,8 & 7,2 & 1,91 & NA \\
\hline \multirow[t]{2}{*}{ Kopf et al $^{3}(2011)^{b}$} & $17,0 \pm 2,0$ & AM $9,2 \pm 1,1$ & - & NA \\
\hline & & $\mathrm{PL} 7,0 \pm 1,0$ & - & \\
\hline Guenther et al $\left.\right|^{8}(2017)^{b}$ & $16,3 \pm 1,6$ & $9,7 \pm 1,4$ & 1,65 & $123,8 \pm 21,5$ \\
\hline Guenther et al $^{20}(2017)^{b}$ & $16,1 \pm 2,6$ & $9,6 \pm 1,5$ & 1,67 & NA \\
\hline Edwards et al $\left.\right|^{21}(2007)^{c}$ & $18 \pm 2$ & $9 \pm 2$ & 2,0 & NA \\
\hline Purnell et al $\left.\right|^{5}(2008)^{c}$ & $10,7 \pm 1,3$ & $7,4 \pm 1,2$ & 1,44 & NA \\
\hline Ferretti et a ${ }^{22}(2012)^{c}$ & $18,1 \pm 2,8$ & $9 \pm 2$ & 2,0 & NA \\
\hline Siebold et al ${ }^{23}(2015)^{c}$ & $12,6 \pm 2,3$ & NA & - & $110,9 \pm 14,7$ \\
\hline Fujimaki et al ${ }^{24}(2016)^{c}$ & $\mathrm{NA}$ & NA & - & $175,8 \pm 64,3$ \\
\hline Tampere et al $^{6}(2017)^{c}$ & NA & NA & - & 159,2 \\
\hline${\text { Iriuchishima et } \text { al }^{4}(2015)^{c}}^{c}$ & NA & NA & - & $133,8 \pm 31,3$ \\
\hline Guenther et al $^{8}(2017)^{d, *}$ & $16,6 \pm 1,3$ & $10,2 \pm 1,0$ & 1,62 & $132,8 \pm 15,7$ \\
\hline Guenther et $\mathrm{al}^{8}(2017)^{\mathrm{d}, * *}$ & $16,7 \pm 1,3$ & $10,4 \pm 0,9$ & 1,60 & $136,7 \pm 15,4$ \\
\hline 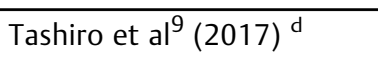 & $15,6 \pm 1,8$ & $14,5 \pm 2,2$ & 1,07 & $182,7 \pm 41,1$ \\
\hline Ichiba et al $^{7}(2014)^{d}$ & $15,2 \pm 1,9$ & NA & - & NA \\
\hline Araki et al ${ }^{10}(2017)^{d}$ & NA & NA & - & $134,7 \pm 22,9$ \\
\hline Kim et $\mathrm{al}^{11}(2018)^{\mathrm{a}}$ & 12,4 & 8,8 & 1,40 & NA \\
\hline Araki et al ${ }^{10}(2017)^{\mathrm{e}}$ & NA & $\mathrm{NA}$ & - & $135,2 \pm 15,1$ \\
\hline Nossos resultados ${ }^{\text {a }}$ & $11,7 \pm 2,0$ & $7,1 \pm 1,4$ & 1,64 & $151,3 \pm 22,29$ \\
\hline
\end{tabular}

Abbreviatlons: AM, anteromedial; NA, não avaliado; PL, posterolateral.

Fonte: Arquivo cedido pela instituição.

aem artroplastias.

bem artroscopias.

'na dissecção de cadáveres.

dpor ressonância magnética.

epor varredura a laser.

*observador 1.

**observador 2.

comprimento da inserção e o peso e altura dos pacientes $(p<0.05)$. A largura e a área de inserção tibial do LCA não foram avaliadas pelos autores.

Park et al ${ }^{12}$ publicaram os resultados das medidas da inserção tibial do LCA feitas em 127 pacientes, durante ATJ. As mensurações foram feitas na margem periférica da inserção ligamentar. O comprimento médio da inserção tibial do LCA foi $13,8 \mathrm{~mm}(10,0-18,0 \mathrm{~mm})$, e a largura média foi de $9,8 \mathrm{~mm}(6,3-13,5 \mathrm{~mm})$. O comprimento tibial $<14 \mathrm{~mm}$ foi encontrado em $53,5 \%$ dos pacientes. Houve correlação significante entre o comprimento da inserção do LCA com o peso e altura dos pacientes. A largura foi significantemente relacionada ao peso, mas a correlação foi fraca.

Em artigo mais recente, Park et al ${ }^{13}$ reportaram os dados prospectivos das mensurações da inserção tibial do LCA realizadas em 209 pacientes, no momento da ATJ, onde também foram demarcadas as margens periféricas da inserção do LCA. Neste estudo, o comprimento médio da inserção tibial do LCA foi de $14,3 \mathrm{~mm}(10,0-19,7 \mathrm{~mm})$, e a largura média foi de $10,2 \mathrm{~mm}(6,3-13,7 \mathrm{~mm})$. O comprimento insercional foi $<14 \mathrm{~mm}$ em $46,5 \%$ da população estudada. A largura da inserção do LCA na tíbia foi associada ao peso, pela regressão linear multivariável.

Nosso estudo apresenta algumas limitações. Embora tenhamos excluído os joelhos com alterações ligamentares morfológicas macroscópicas e aqueles com osteófitos ao redor da inserção tibial do LCA, a idade média de nossa população (69,5 anos) é superior à dos indivíduos que são submetidos à reconstrução cirúrgica do LCA. Nossa amostra pode ser considerada pequena, mas é maior do que as de vários estudos previamente publicados. ${ }^{5,6,22-24}$ Também, a MANOVA foi escolhida para interpretação dos resultados 
exatamente por considerarmos a amostra pequena. Finalmente, todas as mensurações foram feitas por um único membro do nosso grupo, o que pode ser um fator gerador de viés, apesar de terem sido realizadas duas vezes e usados os resultados médios.

\section{Conclusões}

A largura da fóvea tibial do LCA pôde ser prevista pela altura dos pacientes, constituindo um meio de ajudar o cirurgião a escolher o enxerto mais adequado para cada paciente nas reconstruções do LCA.

Conflito de interesses

Os autores declaram não haver conflito de interesses.

\section{Referências}

1 Mall NA, Chalmers PN, Moric M, et al. Incidence and trends of anterior cruciate ligament reconstruction in the United States. Am J Sports Med 2014;42(10):2363-2370

2 van Eck CF, Lesniak BP, Schreiber VM, Fu FH. Anatomic single- and double-bundle anterior cruciate ligament reconstruction flowchart. Arthroscopy 2010;26(02):258-268

3 Kopf S, Pombo MW, Szczodry M, Irrgang JJ, Fu FH. Size variability of the human anterior cruciate ligament insertion sites. Am J Sports Med 2011;39(01):108-113

4 Iriuchishima T, Ryu K, Aizawa S, Fu FH. Proportional evaluation of anterior cruciate ligament footprint size and knee bony morphology. Knee Surg Sports Traumatol Arthrosc 2015;23(11):3157-3162

5 Purnell ML, Larson AI, Clancy W. Anterior cruciate ligament insertions on the tibia and femur and their relationships to critical bony landmarks using high-resolution volume-rendering computed tomography. Am J Sports Med 2008;36(11):2083-2090

6 Tampere T, Van Hoof T, Cromheecke M, et al. The anterior cruciate ligament: a study on its bony and soft tissue anatomy using novel 3D CT technology. Knee Surg Sports Traumatol Arthrosc 2017;25 (01):236-244

7 Ichiba A, Kido H, Tokuyama F, Makuya K, Oda K. Sagittal view of the tibial attachment of the anterior cruciate ligament on magnetic resonance imaging and the relationship between anterior cruciate ligament size and the physical characteristics of patients. J Orthop Sci 2014;19(01):97-103

8 Guenther D, Irarrázaval S, Albers M, Vernacchia C, Irrgang JJ, Musahl V, et al. Area of the tibial insertion site of the anterior cruciate ligament as a predictor for graft size. Knee Surg Sports Traumatol Arthrosc 2017;25(05):1576-1582

9 Tashiro Y, Lucidi GA, Gale T, et al. Anterior cruciate ligament tibial insertion site is elliptical or triangular shaped in healthy young adults: high-resolution 3-T MRI analysis. Knee Surg Sports Traumatol Arthrosc 2018;26(02):485-490

10 Araki D, Thorhauer E, Tashman S. Three-dimensional isotropic magnetic resonance imaging can provide a reliable estimate of the native anterior cruciate ligament insertion site anatomy. Knee Surg Sports Traumatol Arthrosc 2018;26(05):1311-1318

11 Kim SH, Lee HJ, Park YB, Jeong HS, Ha CW. Anterior Cruciate Ligament Tibial Footprint Size as Measured on Magnetic Reso- nance Imaging: Does It Reliably Predict Actual Size? Am J Sports Med 2018;46(08):1877-1884

12 Park YB, Song YS, Kim SC, Park YG, Ha CW. The size of tibial footprint of anterior cruciate ligament and association with physical characteristics in Asian females. Arch Orthop Trauma Surg 2015;135(07):985-992

13 Park YB, Ha CW, Kim HJ, Park YG. Preoperative prediction of anterior cruciate ligament tibial footprint size by anthropometric variables. Knee Surg Sports Traumatol Arthrosc 2017;25(05): 1638-1645

14 Schwartzman S. Fora de foco: diversidade e identidades étnicas no Brasil. Novos Estud CEBRAP 1999;55:83-96

15 Douglas MJ, Hutchison JD, Sutherland AG. Anterior cruciate ligament integrity in osteoarthritis of the knee in patients undergoing total knee replacement. J Orthop Traumatol 2010;11(03): 149-154

16 Lee GC, Cushner FD, Vigoritta V, Scuderi GR, Insall JN, Scott WN Evaluation of the anterior cruciate ligament integrity and degenerative arthritic patterns in patients undergoing total knee arthroplasty. J Arthroplasty 2005;20(01):59-65

17 Mullaji AB, Marawar SV, Simha M, Jindal G. Cruciate ligaments in arthritic knees: a histologic study with radiologic correlation. J Arthroplasty 2008;23(04):567-572

18 Morgan CD, Kalman VR, Grawl DM. Definitive landmarks for reproducible tibial tunnel placement in anterior cruciate ligament reconstruction. Arthroscopy 1995;11(03):275-288

19 Tállay A, Lim MH, Bartlett J. Anatomical study of the human anterior cruciate ligament stump's tibial insertion footprint. Knee Surg Sports Traumatol Arthrosc 2008;16(08):741-746

20 Guenther D, Irarrázaval S, Nishizawa Y, et al. Variation in the shape of the tibial insertion site of the anterior cruciate ligament: classification is required. Knee Surg Sports Traumatol Arthrosc 2017;25(08):2428-2432

21 Edwards A, Bull AM, Amis AA. The attachments of the anteromedial and posterolateral fibre bundles of the anterior cruciate ligament: Part 1: tibial attachment. Knee Surg Sports Traumatol Arthrosc 2007;15(12):1414-1421

22 Ferretti M, Doca D, Ingham SM, Cohen M, Fu FH. Bony and soft tissue landmarks of the ACL tibial insertion site: an anatomical study. Knee Surg Sports Traumatol Arthrosc 2012;20(01):62-68

23 Siebold R, Schuhmacher P, Fernandez F, et al. Flat midsubstance of the anterior cruciate ligament with tibial " $\mathrm{C}$ "-shaped insertion site. Knee Surg Sports Traumatol Arthrosc 2015;23(11): 3136-3142

24 Fujimaki Y, Thorhauer E, Sasaki Y, Smolinski P, Tashman S, Fu FH. Quantitative In Situ Analysis of the Anterior Cruciate Ligament: Length, Midsubstance Cross-sectional Area, and Insertion Site Areas. Am J Sports Med 2016;44(01):118-125

25 Lord BR, El-Daou H, Zdanowicz U, Śmigielski R, Amis AA. The role of fibres within tibial attachments of the ACL in resisting tibial displacements. Knee 2017;24(06):12

26 Hamada M, Shino K, Horibe S, Mitsuoka T, Toritsuka Y, Nakamura $\mathrm{N}$. Changes in cross-sectional area of hamstring anterior cruciate ligament grafts as a function of time following transplantation. Arthroscopy 2005;21(08):917-922

27 LaPrade CM, Smith SD, Rasmussen MT, Hamming MG, Wijdicks CA, Engebretsen L, et al. Consequences of tibial tunnel reaming on the meniscal roots during cruciate ligament reconstruction in a cadaveric model, Part 1: The anterior cruciate ligament. Am J Sports Med 2015;43(01):200-206 